\title{
Carboxy-Terminal Region of a Thermostable CITase from Thermoanaerobacter thermocopriae Has the Ability to Produce Long Isomaltooligosaccharides
}

\author{
Woo Soo Jeong ${ }^{1 \dagger}$, Yu-Ri Kim ${ }^{2 \dagger}$, Seong-Jin Hong ${ }^{2}$, Su-Jeong Choi ${ }^{2}$, Ji-Ho Choi ${ }^{1}$, Shin-Young Park ${ }^{1}$, Eui-Jeon Woo ${ }^{3}$, \\ Young Min Kim ${ }^{2 *}$, and Bo-Ram Park ${ }^{1 *}$ \\ ${ }^{1}$ Department of Agro-Food Resources, National Institute of Agricultural Sciences, Rural Development Administration, Jeonju 55365, \\ Republic of Korea \\ ${ }^{2}$ Department of Food Science and Technology, Chonnam National University, Gwangju 61186, Republic of Korea \\ ${ }^{3}$ Korea Research Institute of Bioscience E Biotechnology (KRIBB), Daejeon 34141, Republic of Korea
}

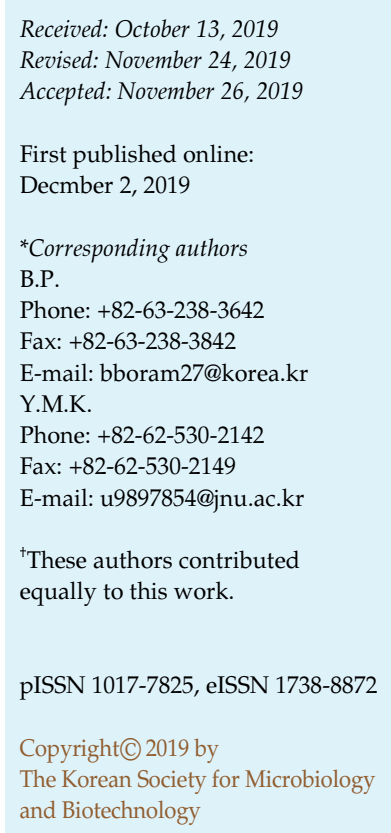

Isomaltooligosaccharides (IMOs) have good prebiotic effects, and long IMOs (LIMOs) with a degree of polymerization (DP) of 7 or above show improved effects. However, they are not yet commercially available, and require costly enzymes and processes for production. The Nterminal region of the thermostable Thermoanaerobacter thermocopriae cycloisomaltooligosaccharide glucanotransferase (TtCITase) shows cyclic isomaltooligosaccharide (CI)-producing activity owing to a catalytic domain of glycoside hydrolase (GH) family 66 and carbohydrate-binding module (CBM) 35. In the present study, we elucidated the activity of the C-terminal region of TtCITase (TtCITase-C; Met740-Phe1,559), including a CBM35-like region and the GH family 15 domain. The domain was successfully cloned, expressed, and purified as a single protein with a molecular mass of $115 \mathrm{kDa}$. TtCITase-C exhibited optimal activity at $40^{\circ} \mathrm{C}$ and $\mathrm{pH} 5.5$, and retained $100 \%$ activity at $\mathrm{pH} 5.5$ after 18 -h incubation. TtCITase-C synthesized $\alpha-1,6$ glucosyl products with over seven degrees of polymerization (DP) by an $\alpha-1,6$ glucosyl transfer reaction from maltopentaose, isomaltopentaose, or commercialized maltodextrins as substrates. These results indicate that TtCITase-C could be used for the production of $\alpha-1,6$ glucosyl oligosaccharides with over DP7 (LIMOs) in a more cost-effective manner, without requiring cyclodextran.

Keywords: Cycloisomaltooligosaccharide glucanotransferase, cyclodextran, long isomaltooligosaccharides, Thermoanaerobacter thermocopriae

\section{Introduction}

Prebiotics are non-digestible carbohydrates that are beneficial for the intestinal microbial balance in the human gut [1] Among the various oligosaccharides, isomaltooligosaccharides (IMOs) comprising 40\% $\alpha-1,6-$ glucosidic linkages have shown promising prebiotic effects [2]. Commercialized IMOs with a degree of polarization (DP) ranging from 2 to 6 and lower numbers of $\alpha-1,6-$ glucosidic linkages were produced from corn starch by $\alpha$ amylase and transglucosidase enzymes [3]. Dextranase has also been used to synthesize pure IMOs via the hydrolysis of dextran with $\alpha$-1,6-glucan linkages, and longer IMOs with DP2-DP10 can be produced from sucrose-only substrates through the combined enzymatic action of dextranase and dextransucrase [4]. Furthermore, dextran dextrinase (DDase), belonging to the glycoside hydrolase (GH) family 15 isolated from Gluconobacter oxydans, was able to produce dextran with a high DP by the successive transfer of $\alpha-1,6$-glucosyl units from maltodextrins [5]. Long IMOs (LIMOs) with DP7 or higher are resistant to hydrolysis by human glycosidases, and are therefore 
absorbed at much lower amounts and are retained in the colon for relatively longer periods [6, 7]. Moreover, LIMOs have been shown to promote quercetin-3-glucoside in the lumen [8, 9] and to improve the production of tumor necrosis factor-alpha in primary macrophages via Toll-like receptor 4 signaling with pro-inflammatory effects [9]. However, it has been a challenge to manufacture IMOs with DP2-DP4 for producing these potentially beneficial LIMOs above DP7.

Cycloisomaltooligosaccharide glucanotransferase (CITase, E.C. 2.4.1.248) is an enzyme belonging to GH family 66 that produces cyclic isomaltooligosaccharides (CIs). CIs are polymers of D-glucose units that are linked via $\alpha-1,6$ linkages with a DP of 7-17 through intra-molecular cyclization from dextran, $\alpha-1,6$-glucan [10-12]. CIs have been reported to exhibit encapsulating and anti-cariogenic activities [13], demonstrating their potential as alternatives for cyclomaltodextrin with limitations in low water solubility. CITase was first reported in Bacillus circulans T-3040 (CITaseT3040) [12] Since then, four kinds of CITases, CITase-598K from Paenibacillus sp. 598K [14], CITase-U155 from Bacillus circulans U-155 [15], PDCITase from Paenibacillus daejeonensis [16], and TtCITase from Thermoanaerobacter thermocopriae [17], have been investigated for their biochemical properties. Among them, CITase-598K [14] and PDCITase showed high activity at an alkaline $\mathrm{pH}$, and TtCITase showed higher thermal stability than the others [17]. Recently, CIs from B. circulans T-3040 and Paenibacillus sp. 598K were produced from starch by co-catalysis with 6- $\alpha$ glucosyltransferase (6-GT) and CITase [18, 19]. At present, CIs are typically produced from dextran by CITase alone or from an $\alpha-1,4$ glucan starch via cooperation between 6-GT and CITase [19].

We previously reported the characterization of a thermostable CITase from $T$. thermocopriae, and showed that the recombinant C-terminal region (Met740-Phe1,559) of TtCITase (TtCITase-C) could hydrolyze maltodextrin with $\alpha-1,4$ - or $\alpha-1,6$-glucosidic linkages, and simultaneously transfer the glucosyl groups via $\alpha-1,6$-glucosidic linkage formation [17]. Based on these findings, we hypothesized that a LIMO with over DP7 could be produced from starch or commercially available maltodextrins by the enzymatic activity of TtCITase-C. This would be a highly economical approach for IMO production, as the typical substrates dextran and sucrose are more expensive than starch. Therefore, co-catalysis by the N-terminal region of TtCITase (from Met1 to Val876), comprising GH family 66 and carbohydrate-binding module (CBM) 35 with CITase activity, and the C-terminal region of TtCITase can be a cost-effective approach to produce CIs from starch or maltodextrin without requiring any dextran.

To validate this possibility, in this study, we performed multiple sequence alignment analysis to predict the function of the C-terminal region of GH family 15, and determined the biochemical properties of TtCITase-C. Further, we analyzed the optimal $\mathrm{pH}$, temperature, stability, and products formed from $\alpha-1,4 / \alpha-1,6$-glucosidic-linked substrates.

\section{Materials and Methods}

\begin{abstract}
Materials
Escherichia coli strains DH5 $\alpha$ and BL21 (DE3) were used for cloning and over-expression of TtCITase-C with the plasmid pGEX-6p-1 (GE Healthcare, USA). E. coli strains were cultured in Luria-Bertani (LB) medium (Difco, BD Biosciences, USA) supplemented with ampicillin $(100 \mu \mathrm{g} / \mathrm{ml})$ at $37^{\circ} \mathrm{C}$. Standards of maltooligosaccharides (MOs; maltose, M2; maltotriose, M3; maltotetraose, M4; maltopentaose, M5) and IMOs (isomaltose, IG2; isomaltotriose, IG3; panose, $\mathrm{P}$; isomaltotetraose, IG4; isomaltopentaose, IG5) were purchased from Megazyme Co. (Copenhagen, Denmark), Carbosynth (Compton, UK), or TCI (Tokyo Chemical Industry, Japan). All other chemicals used in this study were of analytical reagent-grade and purchased from Sigma-Aldrich Chemical Co. (USA).
\end{abstract}

\section{Amino Acid Sequence Analysis}

The TtCITase coding gene sequence (WP_0289926961) with CITase activity was obtained from the National Center for Biotechnology Information (NCBI) database. The putative protein sequence (amino acids 1-1,559) was predicted using BLAST, an algorithm for comparing primary biological sequence information, including amino acid sequences, available online at the NCBI database (http:/ / blast.ncbi.nlm.nih.gov) and Carbohydrate Active Enzyme information available at the CAZy database (http:// www.cazy.org). Multiple sequence alignment of selected amino acids with reported enzyme activity from the GH family 66 and 15 domains, and the truncated -terminal region of TtCITase (TtCITase-C) were carried out using CLUSTAL X 2.1.

\section{Cloning of the Partial CITase Gene from T. thermocopriae}

The TtCITase-C coding gene was amplified by PCR from the genomic DNA of $T$. thermocopriae (RIKEN Bio-resource Center Gene Engineering Division, Japan). The gene was amplified with the following primer pair: forward primer, 5'-GGATCCCCG GAATTCATGATTTATGTAAAGCGTACGATAACCAC-3' and reverse primer, 5'-ATGCGGCCGCTCGAGTTAAAAATCAGGT AATCGTAGATCAAACC-3' (underlines indicate the restriction enzyme sites EcoRI and XhoI, respectively). The amplified gene was cloned in E. coli via the pGEX-6p-1 vector with an N-terminal GST-tag, and confirmed by DNA sequencing analysis at Bionics Inc. (Korea). 
Protein Expression and Purification of Recombinant TtCITase-C

E. coli BL21 (DE3) harboring the TtCITase-C expression vector was grown in LB medium supplemented with $100 \mu \mathrm{g} / \mathrm{ml}$ ampicillin (Georgiachem, USA) until reaching an optical density of 0.5-0.8 at $600 \mathrm{~nm}$. The E. coli cells were then induced by the addition of $0.5 \mathrm{mM}$ isopropyl- $\beta$-D-1-thiogalactopyranoside (Sigma-Aldrich Chemical Co.) at $18^{\circ} \mathrm{C}$ for $18 \mathrm{~h}$. The induced cells were harvested by centrifugation at $6,120 \times g$ for $10 \mathrm{~min}$ and resuspended in lysis buffer (20 mM sodium phosphate buffer $\mathrm{pH} 7.4,1 \mathrm{mM}$ phenylmethylsulfonyl fluoride, $1 \mathrm{mM}$ dithiothreitol, $1 \mathrm{mM}$ EDTA, or $1 \mathrm{mg} / \mathrm{ml}$ lysozyme). The cells were disrupted by sonication (SONICS, $10 \mathrm{~s}, 40 \%$ amplification) at $4^{\circ} \mathrm{C}$ and the cellular debris was removed by centrifugation at $16,200 \times g$ for $10 \mathrm{~min}$. After centrifugation, the crude lysate was passed through a Glutathione Sepharose 4 Fast Flow affinity column (GE Healthcare). The column was washed with $20 \mathrm{mM}$ Tris- $\mathrm{HCl}$ buffer ( $\mathrm{pH}$ 8.0) containing $150 \mathrm{mM} \mathrm{NaCl}$. Subsequently, the recombinant TtCITase-C was eluted with $50 \mathrm{mM}$ Tris- $\mathrm{HCl}$ buffer $(\mathrm{pH}$ 8.0) containing $10 \mathrm{mM}$ reduced glutathione. The protein concentration was determined according to the Bradford method with bovine serum albumin as the standard [20]. The final purified recombinant protein was resolved using $10 \%(\mathrm{w} / \mathrm{v}$ ) sodium dodecyl sulfate-polyacrylamide gel electrophoresis (SDS-PAGE; SMOBIO Technology Inc., Taipei).

\section{Enzyme Assay}

The optimum $\mathrm{pH}$ and temperature of TtCITase-C activity were determined by measuring the release of para-nitrophenol $(p \mathrm{NP})$ from $6 \mathrm{mM}$ para-nitrophenyl- $\alpha$-glucopyranoside ( $p \mathrm{NPG}$ ) as the substrate [21]. The optimum $\mathrm{pH}$ was determined by measuring the enzyme activity at $40^{\circ} \mathrm{C}$ in $120 \mathrm{mM}$ Britton-Robinson buffer (B$\mathrm{R}$ buffer, $\mathrm{pH}$ 2.0-9.0). The optimum temperature was determined in $80 \mathrm{mM}$ B-R buffer (pH 5.5) at different temperatures $\left(30-80^{\circ} \mathrm{C}\right)$. The temperature stability was analyzed by incubating $0.5 \mu \mathrm{g} / \mathrm{ml}$ of the purified enzyme for $1,3,6,12$, or $24 \mathrm{~h}$. To determine the $\mathrm{pH}$ stability of TtCITase-C, the purified enzyme was kept in $100 \mathrm{mM}$ B-R buffer ( $\mathrm{pH}$ 2.0-9.0) at $4^{\circ} \mathrm{C}$ for $18 \mathrm{~h}$, and the residual activity of enzymes from different test conditions at $40^{\circ} \mathrm{C}$ for $15 \mathrm{~min}$ in $120 \mathrm{mM}$ B-R buffer with $6 \mathrm{mM} p \mathrm{NPG}$ was compared.

\section{High-Performance Anion-Exchange Chromatography-Amperometric Detection (HPAEC-PAD) Analysis}

The reaction mixtures containing $5 \mathrm{mM}$ maltopentaose (M5) and isomaltopentaose (IG5) in $120 \mathrm{mM}$ B-R buffer (pH 5.0) with an equal volume of purified TtCITase-C were incubated at $40^{\circ} \mathrm{C}$ for $6 \mathrm{~h}$ and analyzed using an HPAEC system (ICS 5000 Dionex chromatography system, Thermo Fisher Scientific, USA) fitted with a CarboPac PA200 column $(4 \times 250 \mathrm{~mm})$ and PA 200 precolumn $(4 \times 50 \mathrm{~mm})$, and enabled with amperometric detection (HPAEC-PAD). The system was created using a PEEK tube (0.24 mm i.d.), gradient mixer ( $2 \mathrm{~mm})$, ED amperometry cell with a $0.25-\mu$ l channel volume, $\mathrm{pH}-\mathrm{Ag} / \mathrm{AgCl}$ reference electrode, 0.002 in the gasket, and gold electrodes. $\mathrm{NaOH}(100 \mathrm{mM})$ and gradient sodium acetate $(\mathrm{NaOAc})$ were used as the solvent for separating and analyzing constituent saccharides. The NaOAc concentration was gradually increased from 0 to $150 \mathrm{mM}$ during $10-40 \mathrm{~min}$ of the total running time $(50 \mathrm{~min})$. The column temperature was maintained at $40^{\circ} \mathrm{C}$, the injection volume was $10 \mu \mathrm{l}$, and the flow rate was $0.5 \mu \mathrm{l} / \mathrm{min}$. $\mathrm{N}_{2}$ gas was used as the stabilizing gas to inhibit the carbonation of the solvent, and molecular-grade distilled water (Merck Millipore, Germany) was used for the analysis. To examine the reaction pattern of the enzyme, products formed from the substrate ( $5 \mathrm{mM}$ isomaltotriose) during the time course of the reaction were determined. To obtain more accurate data of the $\alpha-1,6$ glucanotransferase activity, the reaction products obtained using $10 \%(\mathrm{w} / \mathrm{v})$ maltose and $10 \%(\mathrm{w} / \mathrm{v})$ dextrin (D.E. 13.0-17.0) with TtCITase-C were compared during $16 \mathrm{~h}$.

\section{Matrix-Assisted Laser Deionization-Time-of-Flight Mass Spectrometry (MALDI-TOF/TOF-MS) Analysis}

The molecular mass of the reaction products of TtCITase- $\mathrm{C}$ with M5 and IG5 was measured by MALDI/TOF-MS. The samples were dissolved in a 2,5-dihydroxybenzoic acid (DHB) matrix composed of a 7:3 (v/v) solution of DHB in $0.1 \%(\mathrm{v} / \mathrm{v})$ trifluoroacetic acid/acetonitrile (1:1, v/v) and $10 \mathrm{mM} \mathrm{NaCl}$. The sample was mixed with the matrix solution directly on the MALDI target and was vacuum-dried. MALD-TOF mass spectra were recorded and high-resolution MS data were obtained on a Bruker Ultra flexIII TOF/TOF-MS spectrometer. The data were analyzed with Flex Control 3.0 (Bruker Daltonics, USA). MS data were analyzed in positive reflection mode and spectra were acquired using a reflection mirror. The ion source voltage was $25.00 \mathrm{kV}$ and the detectable $\mathrm{m} / \mathrm{z}$ range was $100-7,000$.

\section{Results and Discussion}

\section{Multiple Sequence Alignment of the T. thermocopriae CITase Gene}

Multiple sequences of CITases were aligned and analyzed to predict the enzymatic function of the $\mathrm{N}$ - or C-terminal region of TtCITase using the protein BLAST (BLASTp) search. BLASTp analysis revealed that the N-terminal region (from Met1 to Val876) of TtCITase had high amino acid sequence homology (53.9-56.8\%) with four previously reported CITases (CITase-T3040, CITase-598K, CITaseU155, and PDCITase). Moreover, TtCITase showed high amino acid sequence homology (36\%) with thermostable dextranase (TpDex), a putative dextranase from Thermoanaerobacter pseudethanolicus belonging to GH family 66. This was an expected result given that these CITases originated from the same genus; moreover, CITase and endodextranase both belong to GH family 66. The CBM35 in the $\mathrm{N}$-terminal region of TtCITase is important for CITase activity [22]. When compared with the other CITases, TtCITase can be used to produce relatively large CIs at 


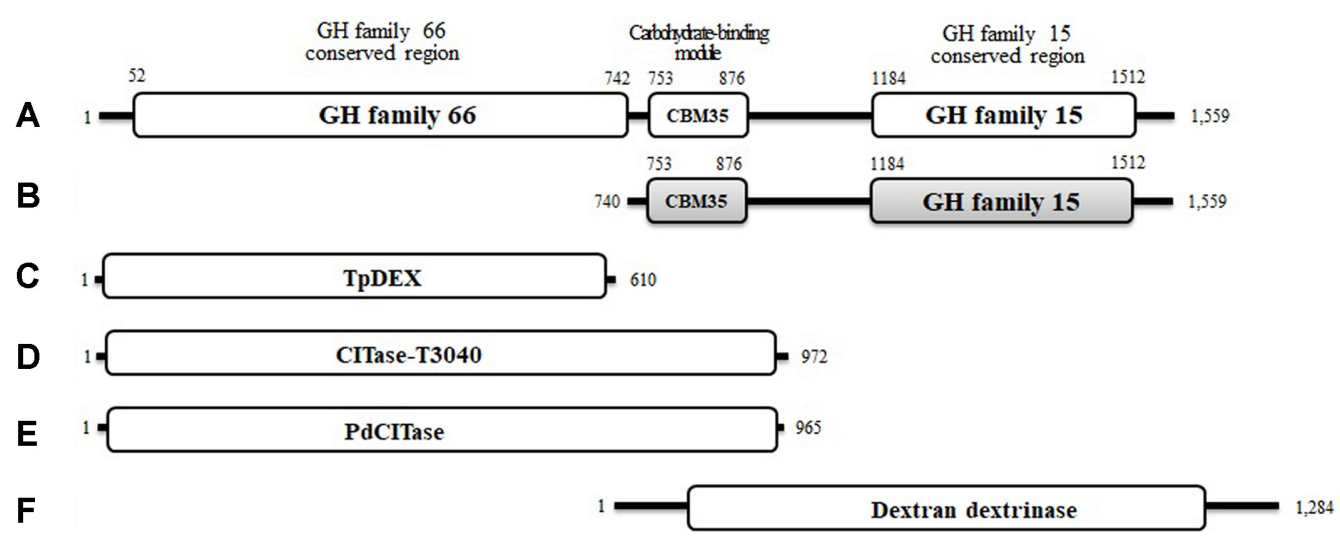

Fig. 1. Schematic diagram of the carbohydrate-binding protein catalytic domain of cycloisomaltooligosaccharide glucanotransferases (CITases).

Aligned amino acid sequences were derived from (A) thermostable CITase of Thermoanaerobacter thermocopriae (TtCITase; GH family 66, CBM35, and GH family 15), (B) the carboxy-terminal of thermostable CITase from T. thermocopriae (TtCITase-C; CBM35 and GH family 15), (C) thermostable dextranase from Thermoanaerobacter pseudethanolicus (TpDEX, GH family 66), (D) CITase from Bacillus circulans T-3040 (CITaseT3040, GH family 66), (E) CITase from Paenibacillus daejeonesis (PDCITase, GH family 66), and (F) dextran dextrinase from Gluconobacter oxydans (GoDDase, GH family 15).

high temperatures [17]. Interestingly, the 819 amino acid Cterminal region (from Met740 to Phe1,559) of TtCITase without CBM35 (TtCITase-C) showed high amino acid sequence homology (31\%) with the DDase from G. oxydans of GH family 15 (Fig. 1). These results suggested that TtCITase may have dual functions, with the N-terminal region acting like other members of GH family 66 and the C-terminal region acting like the DDase of GH family 15 linked by CBM35.

\section{Cloning and Expression of TtCITase-C}

TtCITase is composed of CITase, a CBM35-like region, and a DDase-like region. We previously reported the functional properties of thermostable CITase [8]. However, when we cloned the C-terminal region alone, no enzymatic activity was detected (data not shown). Therefore, we cloned and stably expressed the partial gene sequence of TtCITase-C (Met740-Phe1,559 of TtCITase) encoding the Cterminal region that included the CBM 35-like region (Fig. 2). The purified TtCITase-C showed a high yield $(24 \%)$ and specific activity $(0.024 \mathrm{U} / \mathrm{mg})$, and a molecular weight was $115 \mathrm{kDa}$ as determined by SDS-PAGE analysis (Fig. 2).

\section{Effects of $\mathrm{pH}$ and Temperature on TtCITase-C Activity and Stability}

Next, we determined the effects of different $\mathrm{pH}$ and temperatures on the activity of purified TtCITase-C. TtCITase-C exhibited the highest activity at pH 5.5 (Fig. 3A), and the enzyme was stable within only a narrow $\mathrm{pH}$ range ( $\mathrm{pH}$ 5.0-5.5). The activity of TtCITase-C dropped to 74\% at $\mathrm{pH} 7.0,50 \%$ at $\mathrm{pH} 6.5$, and was lower than $10 \%$ at $\mathrm{pH} 8.0$ (Fig. 4A). Thus, the optimal $\mathrm{pH}$ for TtCITase-C activity differs from that reported for other CITases, including TtCITase (pH 6.0), CITase-T3040 (pH 5.5-8.0), and PDCITase ( $\mathrm{pH}$ 8.0) $[11,13,23]$. The optimum temperature for TtCITase$\mathrm{C}$ activity was found to be $40^{\circ} \mathrm{C}$ with $100 \%$ enzyme activity observed at $30^{\circ} \mathrm{C}$ and $40^{\circ} \mathrm{C}$ for $24 \mathrm{~h}$; however, the activity

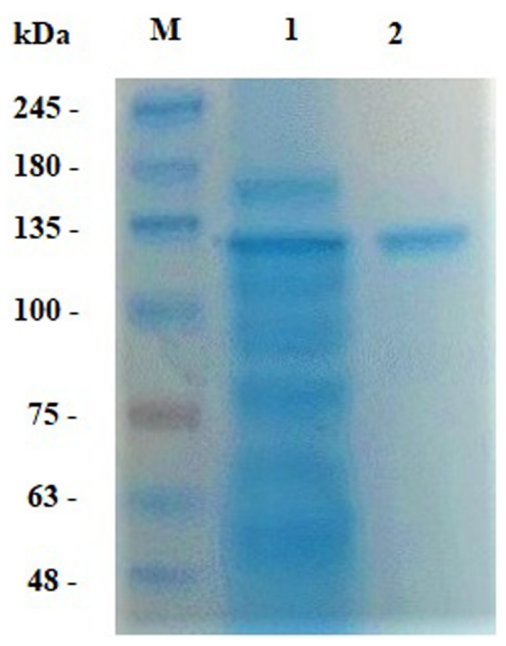

Fig. 2. SDS-PAGE gel of the expression of purified TtCITase-C. Lane M, protein molecular weight marker $(48,63,75,100,135$, 180, or $245 \mathrm{kDa}$ ); Lane 1, cell lysate supernatant of E. coli cells expressing TtCITase-C; Lane 2, purified TtCITase-C by a GST affinity column. 
A

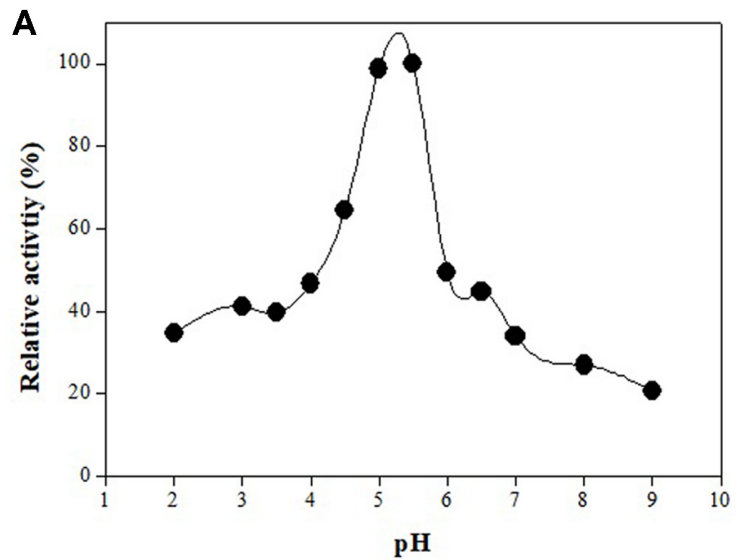

B

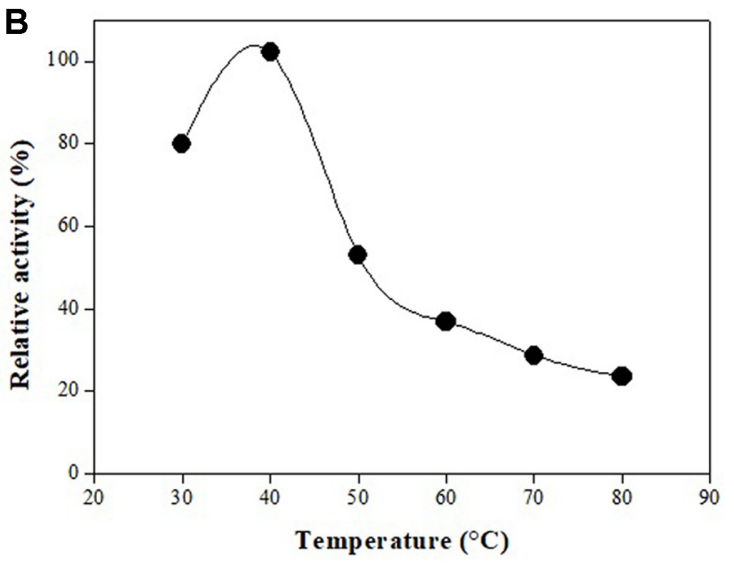

Fig. 3. Optimum $\mathrm{pH}(\mathbf{A})$ and temperature (B) for TtCITase-C activity.

sharply decreased over $50^{\circ} \mathrm{C}$ (Figs. 3B and 4B). These results were similar to those reported for TtCITase $\left(60^{\circ} \mathrm{C}\right)$, CITase-T3040 $\left(40^{\circ} \mathrm{C}\right)$, and PDCITase $\left(40^{\circ} \mathrm{C}\right)$. The significant homology observed between the full sequence of TtCITase (53.9-56.8\%) and these various other CITases could explain their similar optimum $\mathrm{pH}$. Although TtCITase showed thermostable enzymatic properties, TtCITase-C showed inferior thermostability and reduced activity at higher temperatures. The thermophilic dextranase from $T$. pseudethanolicus, used for the structural comparison with other mesophilic enzymes, is truncated at the exposed loop region, thereby leaving many hydrophilic amino acid residues exposed at the surface [24]. Since charged residues can result in stable ion pairs that contribute to protein thermostability $[23,24]$, we presume that TtCITase-C was less stable at $50^{\circ} \mathrm{C}$ and above due to the lack of the $\mathrm{N}$ terminus that contributes to the thermal stability, such as TtDEX (Fig. 1).

\section{Analysis of Reaction Products}

To determine the hydrolysis and glucosyl transfer activity of TtCITase-C, we performed a time-course analysis of the products formed from the substrates maltopentaose and isomaltopentaose. TtCITase-C produced G, IG2, IG4, and IG5 as the reaction products of transglucosylation from IG3 as the substrate in the early stage of the reaction (Fig. 5). Subsequently, the level of IG3 decreased rapidly over time, and the amount of IG2 (estimated by the relative area) exceeded that of IG3 after $1 \mathrm{~h}$. IG2, generated from IG3, IG4, and IG5, was hydrolyzed and thus its level slightly
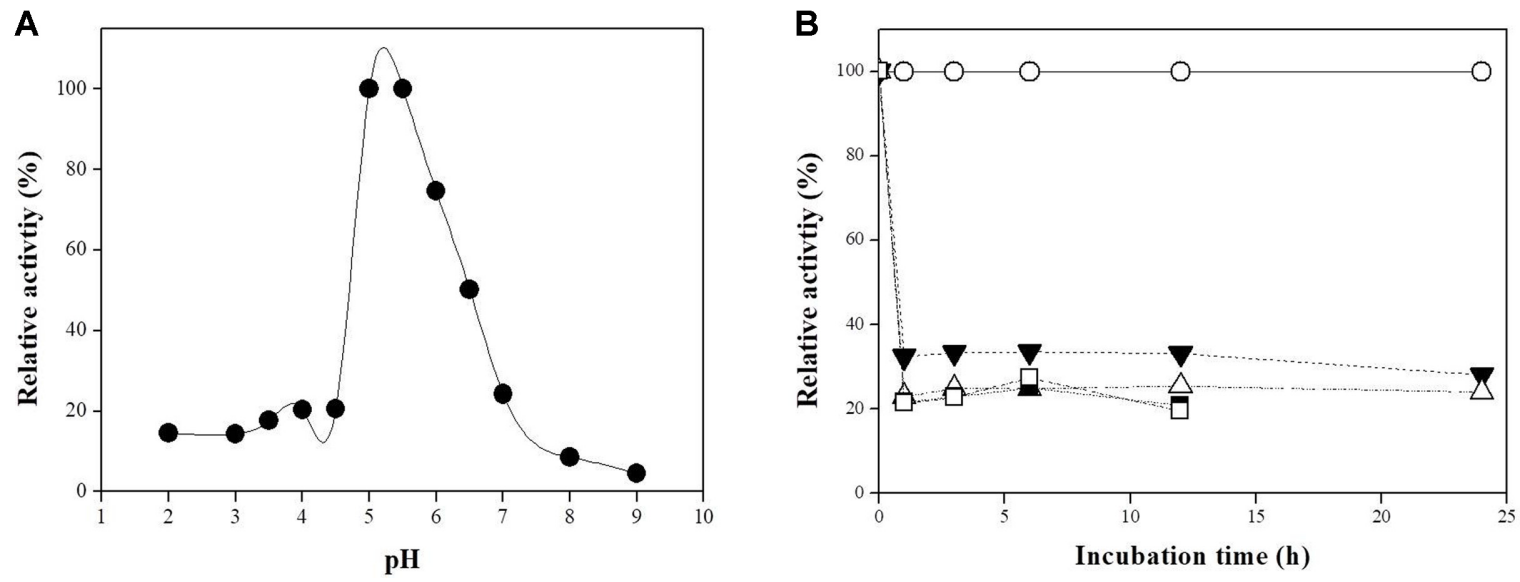

Fig. 4. The $\mathrm{pH}(\mathbf{A})$ and temperature (B) stability of TtCITase-C.

The purified TtCITase- $\mathrm{C}$ was incubated for various lengths of time and residual enzyme activity was measured at $40^{\circ} \mathrm{C}$ in Britton-Robinson buffer (pH 5.5). $\bullet, 30^{\circ} \mathrm{C} ; \bigcirc, 40^{\circ} \mathrm{C} ; \boldsymbol{\nabla}, 50^{\circ} \mathrm{C} ; \triangle, 60^{\circ} \mathrm{C} ; \mathbf{\square}, 70^{\circ} \mathrm{C} ; \square, 80^{\circ} \mathrm{C}$. 


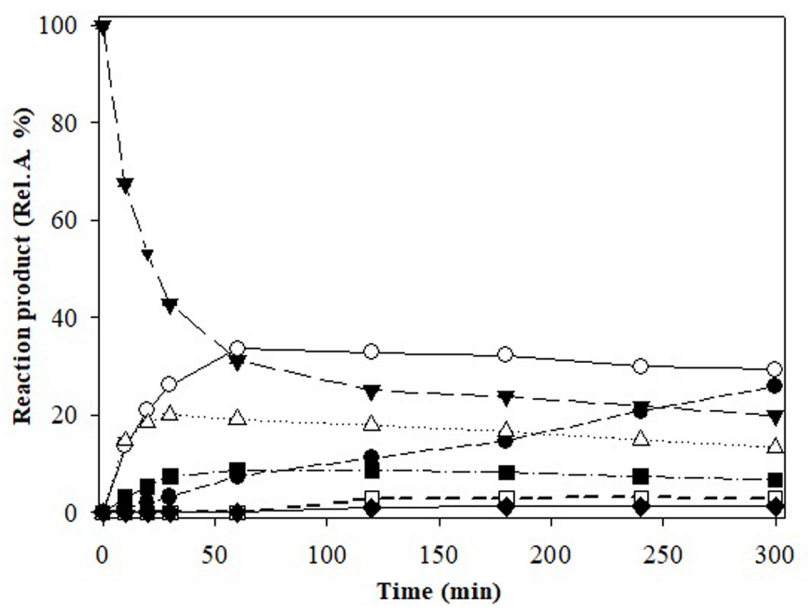

Fig. 5. Dynamics of reaction products during the reaction of TtCITase-C with $5 \mathrm{mM}$ isomaltotriose at $40^{\circ} \mathrm{C}$ for $5 \mathrm{~h}$.

-, Glucose (G); $\bigcirc$, isomaltose (IG2); $\nabla$, isomaltotriose (IG3); $\triangle$, isomaltotetraose (IG4); $\mathbf{\square}$, isomaltopentaose (IG5); $\square$, isomaltohexaose $(\mathrm{IG} 6) ; \diamond$, isomalotheptaose (IG7).

decreased after $2 \mathrm{~h}$, whereas IG6 and IG7 were produced in small amounts over the same time period. The amount of glucose, which is a by-product of the transglucosylation and hydrolysis of IMOs, was higher than the remaining amount of IG3 expected according to the relative area of the chromatogram peak.

The production of M5 and IG5 was assessed via HPAEC analysis. TtCITase-C produced IMOs up to DP8 and DP14 from M5 and IG5, respectively. Because of the dual actions of TtCIT-C in the hydrolysis and transglucosylation with G5, the hydrolyzed glucose residue of the maltooligosyl $\alpha$ 1,4-linkage (donor) would be transferred to the $6 \mathrm{C}-\mathrm{OH}$ of the non-reducing end of another maltooligosaccharide (acceptor), thereby forming the $\alpha-1,6$ linkage (G-1,6-G5). The hydrolysis reaction was repeated three or four times, and G2 and G were produced as residual products. The panose-type materials mixed with $\alpha-1,6 / 1,4$ linkages were also produced as part of the intermediate reaction products of the transglycosylation to maltose. In the same manner, we expected that IG5 would be elongated by the glucosyltransferase activity of the glucose residue at the non-reducing end from the other IG5. Indeed, the reaction of IG5 showed a novel peak, which is presumed to be a LIMO (Fig. 6D).

MALDI-TOF MS identified the IMOs of DP8 to 14 produced by TtCITase-C as $\alpha-1,6$ glucosyl-transferring products. The molecular masses of $\alpha-1,6$-linked D-glucose products from M5 were confirmed to be 364.92 (DP2),

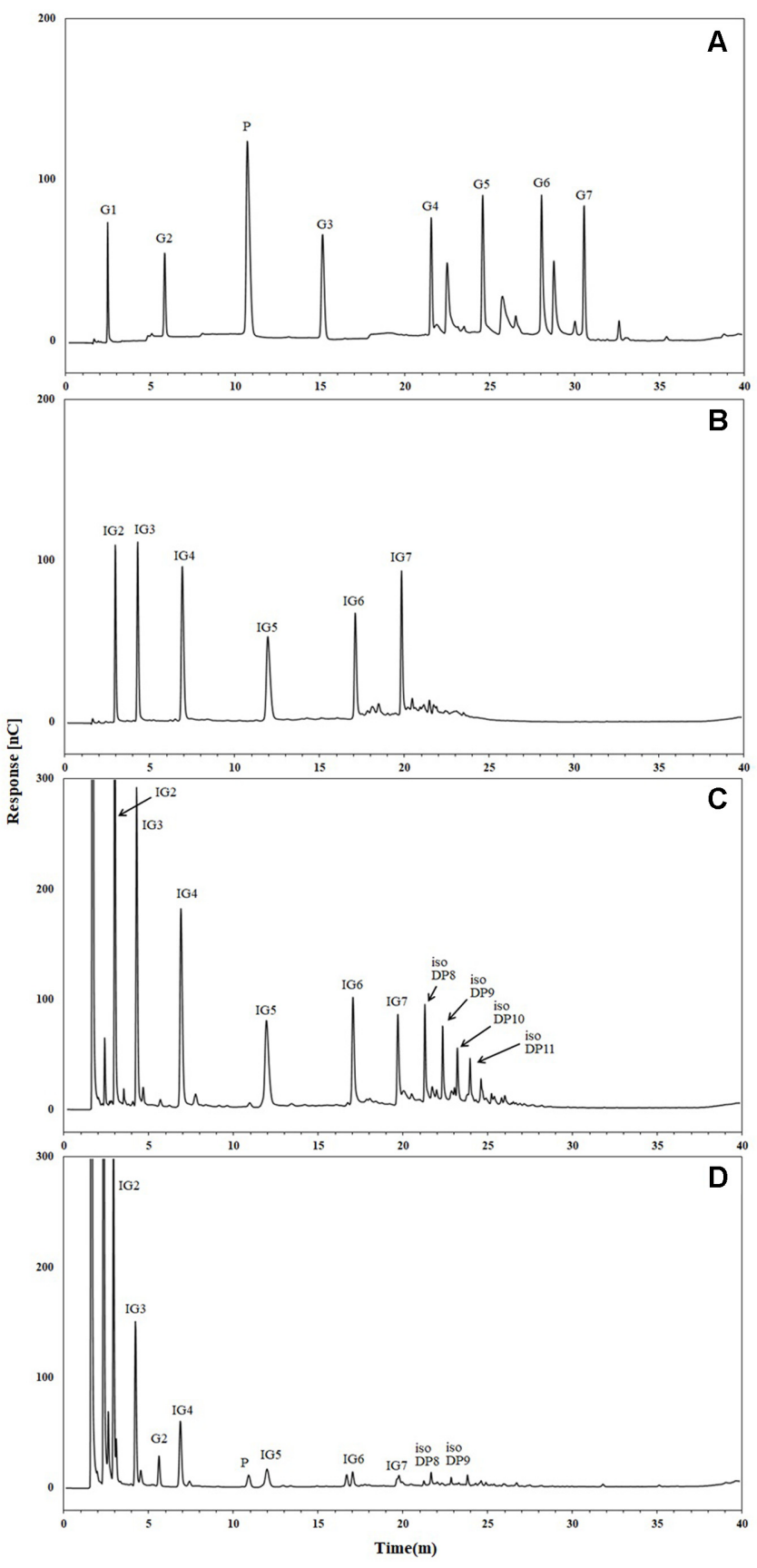

Fig. 6. HPAEC analysis of the product obtained from the reaction of TtCITase- $\mathrm{C}$ with maltopentaose and isomaltopentaose. (A) Maltooligosaccharides (G-G7) and panose (P) standards. (B) Isomaltosaccharides standards (IG2-IG7). (C) Reaction products of TtCITase- $\mathrm{C}$ with maltopentaose. (D) Reaction products of TtCITase-C with isomaltopentaose.

527.03 (DP3), 689.12 (DP4), 851.20 (DP5), 1,013.26 (DP6), 1,172.32 (DP7), 1,337.37 (DP8), and 1,499.42 (DP9) of mass value $(\mathrm{m} / \mathrm{z})($ Fig. $7 \mathrm{~A})$, whereas the reaction product from 
A

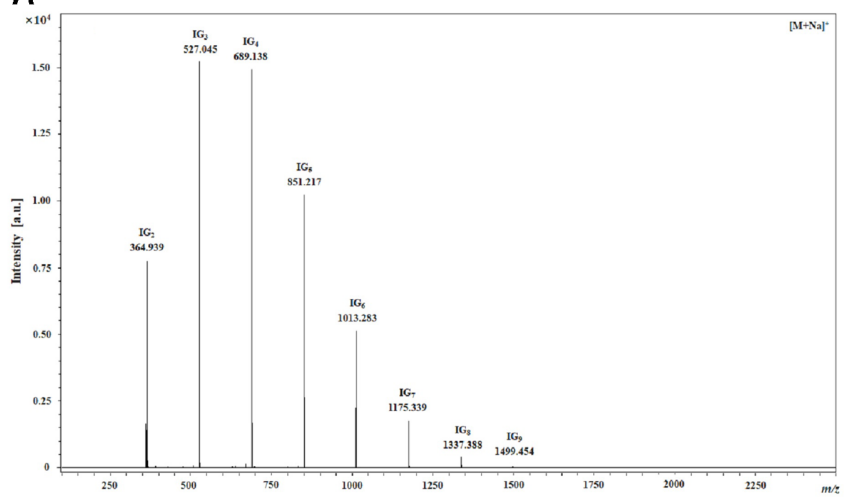

B

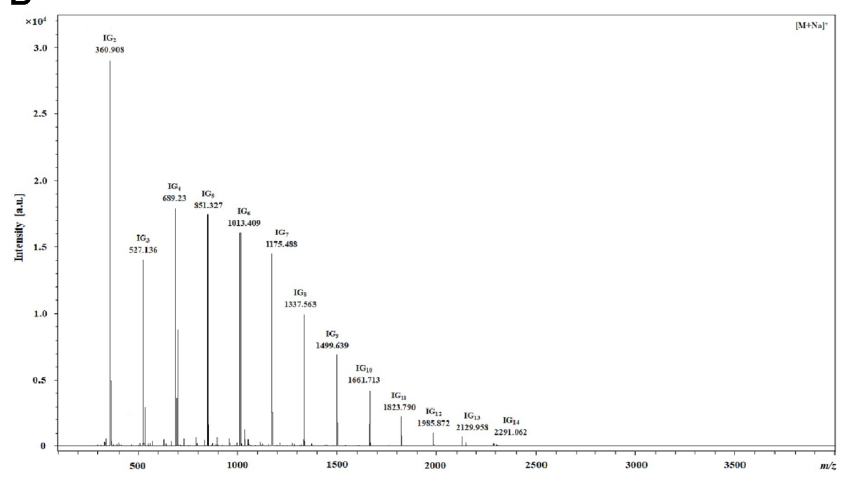

Fig. 7. MALDI-TOF/TOF MS analysis of the reaction products of TtCITase-C with maltopentaose and isomaltopentaose.

(A) Reaction products $\mathrm{TtCITase}-\mathrm{C}$ with maltopentaose. (B) Reaction products of TtCITase-C with isomaltopentaose.

IG5 contained peaks up to 2,291.062 (DP14) (Fig. 7B). In addition to MOs with DP, panose with a-1,6 glucosyl linkages was produced from M2 (Fig. 8C). Maltodextrin with a dextrose equivalent (D.E.) of 13.0-17.0 produced more complicated $\alpha-1,6$ glucosyl-transferring products with longer DPs (Fig. 8D).

The $\alpha$-glucosidase (transglucosidase) from Aspergillus niger was shown to rapidly transfer glucosyl residues to maltooligosaccharides; both the $\alpha-1,4$-glucosidic and $\alpha-1,6$ glucosidic linkages were gradually hydrolyzed at the nonreducing ends, and transformed into smaller molecules of mainly $\alpha-1,6$-glucosidic linkages [25]. TtCITase-C showed homology with DDase belonging to GH family 15 and showed strong $\alpha-1,6$ glucosyltransferase activity, similar to DDase from $G$. oxydans [26] and $\alpha$-glucosidase from A. niger [2]. DDase produced dextran as the main product and a small amount of IMOs, while $A$. niger $\alpha$-glucosidase produced IMOs with low DPs (2-6). However, in the present study, we did not detect the production of dextran or other high-DP products. Moreover, the $\alpha-1,6$ glucosyl-

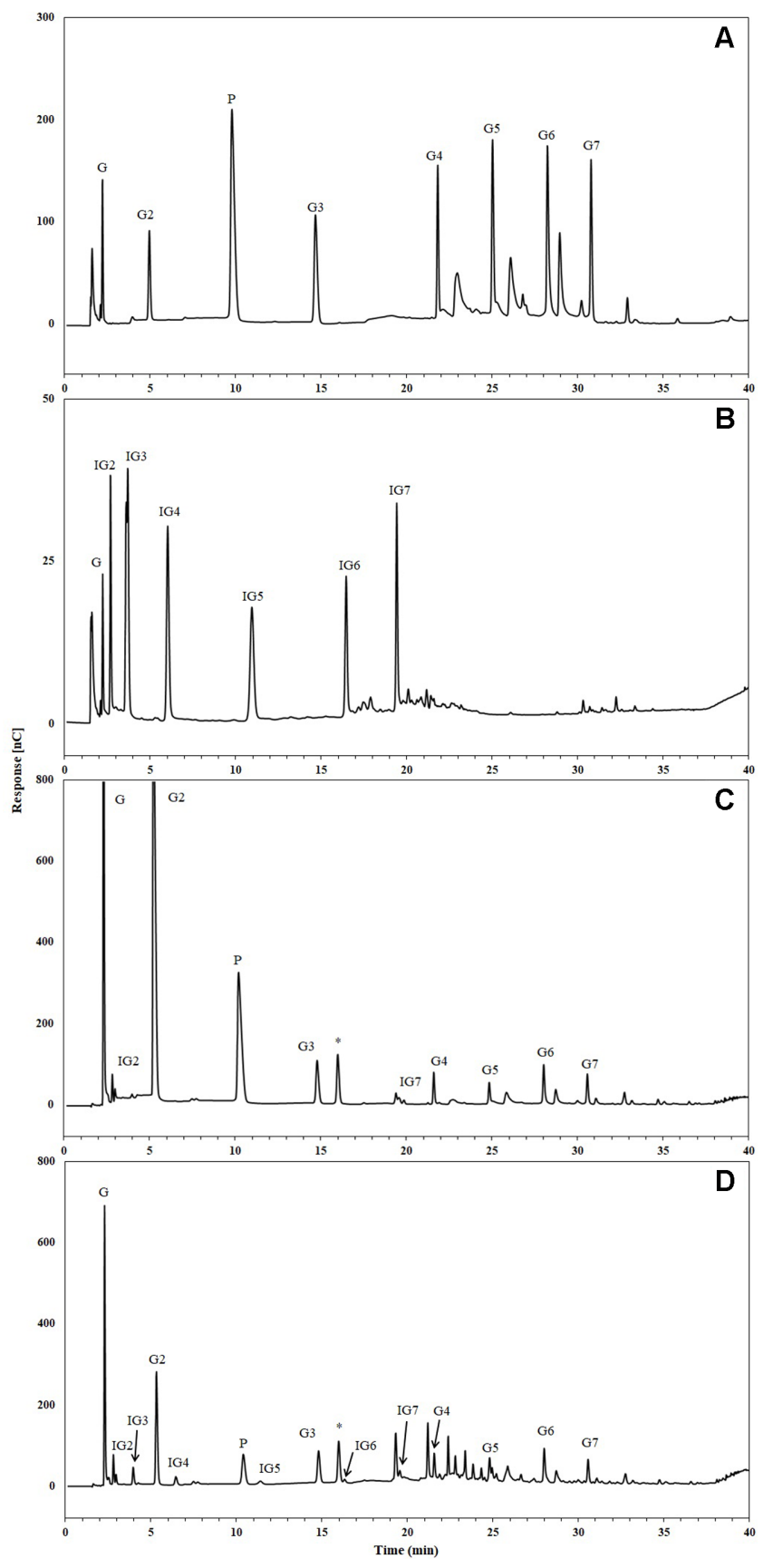

Fig. 8. HPAEC analysis of the product obtained from the reaction of TtCITase-C with maltose and maltodextrin(D.E 13.0-17.0).

(A) Maltooligosaccharides (G-G7) and panose (P) standards. (B) IG2IG7 standards. (C) Reaction products of TtCITase-C with maltose. (D) Reaction products of TtCITase-C with maltodextrin (D.E 13.0-17.0).

transferase enzyme activity of TtCITase-C was intermediate between the $\alpha$-glucosidase activity reported from $A$. niger and the DDase activity from G. oxydans. Further, TtCITase- 
C produced IMOs (DP2-14) and LIMO-like products from substrates with $\alpha$-1,4-glucosidic and $\alpha$-1,6-glucosidic linkages.

In conclusion, this study confirmed that TtCITase-C has enzymatic properties suitable for producing LIMOs with various DPs. Further studies are required to elucidate the three-dimensional structure of TtCITase or TtCITase-C for more accurate classification of the observed enzymatic activity.

\section{Acknowledgements}

This study was supported by the Cooperative Research Program for Agriculture Science and Technology Development (Project No. PJ01312601) Rural Development Administration, Republic of Korea.

\section{Conflict of Interest}

The authors have no financial conflicts of interest to declare.

\section{References}

1. Gibson GR, Roberfroid MB. 1995. Dietary modulation of the human colonic microbiota: Introducing the concept of prebiotics. J. Nutr. 125: 1401-1412.

2. Crittenden RG, Playne MJ. 1996. Production, properties and applications of food-grade oligosaccharides. Trends Food Sci. Technol. 7: 353-361.

3. Kim Y-M, Seo M-Y, Kang H-K, Atsuo K, Kim D. 2009. Construction of a fusion enzyme of dextransucrase and dextranase: application for one-step synthesis of isomaltooligosaccharides. Enzyme Microb. Technol. 44: 159-164.

4. Sadahiro J, Mori H, Saburi W, Okuyama M, Kimura A. 2015. Extracellular and cell-associated forms of Gluconobacter oxydans dextran dextrinase change their localization depending on the cell growth. Biochem. Biophys. Res. Commun. 456: 500505.

5. Debnam ES, Denholm EE, Grimble GK. 1998. Acute and chronic exposure of rat intestinal mucosa to dextran promotes SGLT1-mediated glucose transport. Eur. J. Clin. Invest. 28: 651-658.

6. Kaneko $\mathrm{T}$, Kohmoto $\mathrm{T}$, Kikuchi $\mathrm{H}$, Shiota $\mathrm{M}$, Iino $\mathrm{H}$, Mitsuoka T. 1994. Effects of isomaltooligosaccharides with different degrees of polymerization on human fecal bifidobactcria. Biosci. Biotechnol. Biochem. 58: 2288-2290.

7. Shinoki A, Lang W, Thawornkuno C, Kang HK, Kumagai Y, Okuyama M, et al. 2013. A novel mechanism for the promotion of quercetin glycoside absorption by megalo $\alpha$ 1,6-glucosaccharide in the rat small intestine. Food Chem. 136: 293-296.
8. Hara H, Kume S, Iizuka T, Fujimoto Y, Kimura A. 2018 Enzymatically synthesized megalo-type isomaltosaccharides enhance the barrier function of the tight junction in the intestinal epithelium. Biosci. Biotechnol. Biochem. 82: 629-635.

9. Joe GH, Andoh M, Shinoki A, Lang W, Kumagai Y, Sadahiro J, et al. 2016. Megalo-type $\alpha$-1,6-glucosaccharides induce production of tumor necrosis factor $\alpha$ in primary macrophages via toll-like receptor 4 signaling. Biomed. Res. 37: 179-186.

10. Oguma T, Horiuchi T, Kobayashi M. 1993. Novel cyclic dextrins, cycloisomaltooligosaccharides, from Bacillus sp. T3040 culture. Biosci. Biotechnol. Biochem. 57: 1225-1227.

11. Funane K, Terasawa K, Mizuno Y, Ono H, Miyagi T, Gibu $\mathrm{S}$, et al. 2007. A novel cyclic isomaltooligosaccharide (cycloisomaltodecaose, CI-10) produced by Bacillus circulans T-3040 displays remarkable inclusion ability compared with cyclodextrins. J. Biotechnol. 130: 188-192.

12. Funane K, Terasawa K, Mizuno Y, Ono H, Gibu S, Tokashiki T, et al. 2008. Isolation of Bacillus and Paenibacillus bacterial strains that produce large molecules of cyclic isomaltooligosaccharides. Biosci. Biotechnol. Biochem. 72: 32773280.

13. Kobayashi M, Funane K, Oguma T. 1995. Inhibition of dextran and mutan synthesis by cycloisomaltooligosaccharides. Biosci. Biotechnol. Biochem. 59: 1861-1865.

14. Suzuki R, Terasawa K, Kimura K, Fujimoto Z, Momma M, Kobayashi M, et al. 2012. Biochemical characterization of a novel cycloisomaltooligosaccharide glucanotransferase from Paenibacillus sp. 598K. Biochim. Biophys. Acta 1824: 919-924.

15. Oguma T, Kitao S, Kobayashi M. 2014. Purification and characterization of cycloisomaltooligosaccharide glucanotransferase and cloning of cit from Bacillus circulans U-155. J. Appl. Glycosci. 61: 93-97.

16. Yang S-J, Ko J-A, Kim H-S, Jo M-H, Lee H-N, Park B-R, et al. 2018. Biochemical characterization of alkaliphilic cyclodextran glucanotransferase from an alkaliphilic bacterium, Paenibacillus daejeonensis. J. Microbiol. Biotechnol. 28: 2029-2035.

17. Yang S-J, Choi S-J, Park B-R, Kim Y-M. 2019. Thermostable CITase from Thermoanaerobacter thermocopriae shows negative cooperativity. Biotechnol. Lett. 41: 625-632.

18. Funane $K$, Ichinose $H$, Araki M, Suzuki R, Kimura K, Fujimoto Z, et al. 2014. Evidence for cycloisomaltooligosaccharide production from starch by Bacillus circulans T3040. Appl. Microbiol. Biotechnol. 98: 3947-3954.

19. Ichinose H, Suzuki R, Miyazaki T, Kimura K, Momma M, Suzuki N, et al. 2017. Paenibacillus sp. 598K 6- $\alpha-$ glucosyltransferase is essential for cycloisomaltooligosaccharide synthesis from $\alpha-(1 \rightarrow 4)$-glucan. Appl. Microbiol. Biotechnol. 101: 4115-4128.

20. Bradford MM. 1976. A rapid and sensitive method for the quantitation of microgram quantities of protein utilizing the principle of protein-dye binding. Anal. Biochem. 72: 248-254. 
21. Mao X, Wang S, Kan F, Wei D, Li F. 2012. A novel dextran dextrinase from Gluconobacter oxydans DSM-2003: Purification and properties. Appl. Biochem. Biotechnol. 168: 1256-1264.

22. Suzuki N, Fujimoto Z, Kim YM, Momma M, Kishine N, Suzuki R, et al. 2014. Structural elucidation of the cyclization mechanism of $\alpha-1,6$-glucan by Bacillus circulans T-3040 cycloisomaltooligosaccharide glucanotransferase. J. Biol. Chem. 289: 12040-12051.

23. Mitchell F, Miles S, Neres J, Bichenkova E, Bryce R. 2010. Tryptophan as a molecular shovel in the glycosyl transfer activity of Trypanosoma cruzi trans-sialidase. Biophys. J. 98: L38.
24. Suzuki N, Kishine N, Fujimoto Z, Sakurai M, Momma M, Ko JA, et al. 2015. Crystal structure of thermophilic dextranase from Thermoanaerobacter pseudethanolicus. J. Biochem. 159: 331339.

25. Ota M, Okamoto T, Wakabayashi H. 2009. Action of transglucosidase from Aspergillus niger on maltoheptaose and [U-13C] maltose. Carbohydr. Res. 344: 460-465.

26. Yamamoto K, Yoshikawa K, Okada S. 1993. Structure of dextran synthesized by dextrin dextranase from Acetobacter capsulatus ATCC 11894. Biosci. Biotechnol. Biochem. 57: 14501453. 